\title{
"LA MUSIQUE DEVIENT CE QU'ELLE EST": DYNAMIQUE MUSICALE ET TRACES MATÉRIELLES
}

Rapha\&] Brunner

L'un des éléments sur lesquels se fondait la critique adornienne de la musique de Stravinsky relevait de cette fameuse "pseudomorphose de la musique sur la peinture»,' laquelle allait lui permettre non seulement de mettre l'accent sur le statisme de la musique du compositeur russe, mais aussi d'étendre cette lecture a la production musicale du second après-guerre. En effet, dans sa réactualisation de la critique stravinskienne, datant des années 60 , le philosophe notait la proximité que lui paraissait entretenir la musique récente avec la musique de Stravinsky, bien qu'elle semblât dériver plus directement de celle de Schönberg: ${ }^{2}$ là aussi, la musiqư ne serait plus en mesure de satisfaire l' "obligation qu'elle a de devenir". ${ }^{3}$

Un bref regard sur la situation actuelle, tant du côté des pratiques compositionnelles que du côté de leur appropriation musicologique, inciterait à poursuivre une telle réllexion. Dans le moment des approches de la narrativité et de la perception musicales, où le réel viendrait, comme du delors, au secours de ce devenir, on serait ainsi tenté d'appeler ici une approche dans le cadre non seulement d'un concept viable de l'historicité, mais aussi d'un concept d'ouvre réactualisés, eu égard a une situation épistémologique particulière.

Confrontée aux apories de la réception esthétique de la musique composée au cours du second après-guerre, l'attention du musicologue semble en effet se déplacer fréquemment hors de la dynamique musicale. Elle va parfois jusqu'à porter de manière exclusive en aval de l'osuvre, sur l'institutionnalisation du fait musical en des cadres de réception fonctionnalisís, en amont de l'cuvre, sur la reproduction du cadre précompositionnel, voire sur l'établissement de contraintes cognitives précompositionnelles.

Il ne paraît cependant pas impensable - mỉme si l'opération encourt les risques inluérents a toute approche esthétique, aujourd'hui- de procéder à quelques conciliations el réappropriations. Le soupçon structuraliste qui a pesé sur l'herméneutique, et notamment sur le discours musicologique traditionnel, n'a pas seulement conduit, d'une part au renforcement de l'artefact de l'immanence de l'auuve, d'autre part aux approches périphériques que je viens d'évoquer, mais il a pu également conduire, dans des développements plus récents, à déplacer l'attention portée à la réception de l'auvre vers la réception de son activité génétique.

Si les mises en perspective portant sur la modernité musicale tirent traditionnellement leur légitimité de l'appréhension d'un sujet se positionnant par rapport au donné historique, l'interprétation procéderait donc bien plus de la lecture d'un devenir formel et langagier au sein de l'auvre inusicale elle-mëme; elle s'attacherait moins à l'approche de l' «intention créatrice», à laquelle demeure lice la musicologie traditionnelle, qu'à l'aintention de l'auvre». En d'autres termes, il conviendrait d'approcher ici la modernité du fait musical moins comme la métaphore d'un sujet réngissant au travers de son auvre à une situation de problème que comme la métaphore d'une écriture demeurant alle-même aux prises avec la réalisation maté-

1 Tlizodor WV. Adorno, Philosophie de la noinclle musique (1958), Paris, Gallimard, 1962, pp. 196-97.

2 Theodor W. Adomo, "Stravinsky: Une image dialectipue» in Quasi ma fantasia (1963). Paris. Gallimard. 1982, p. 164.

3 Theodor IV. Adorno, "Vers la musiçue informelle" in Quasi uma fantasia, p. 317. 
rielle - ce serait une mise en perspective herméneutique assumant le soupcon porté par le structuralisme.

L'œuvre musicale moderne n'appelle donc pas seulement l'actualisation d'un sens, reçu mais dès lors esthétiquement figé, elle convoque également le geste qui la produit, sans que ce dernier ne parvienne jamais à s'y fixer, actualisant ainsi un devenir -sa structure de signification propre, pour parler avec la sémiotique, s'étant affranchie d'une structure communicationnelle stable et normée. En effet, une fois émancipée des contraintes langagières, liées à la convention, l'cuvre musicale a produit un eflort incommensurable pour assumer son autonomie, notamment par la théâtralisation de sa propre activité langagière, de son propre devenir, qui peut aller parfois, comme le note Adorno, jusqu'à la critique du langage, voire au sacrifice de l'art. ${ }^{4}$

Pour utiliser des formules éloquentes, il s'agit moins de décrire comment l'auvre uest reçue» que de décrire comment elle "se reçoit» elle-même. Et heureusement, l'œuvre se reçoit mal, ne se recopie pas sans ratures - pour ainsi dire. L'approche de la dynamique musicale à partir des traces matérielles que l'œuvre laisse en amont d'elle-même lui restituerait donc une historicité, quoique, une fois encore, il n'y ait pas lieu de céder au confort des données vérifiables. Pour nécessaire qu'elle puisse être, cette soidisant objectivité n'en constituerait pas moins un leurre: elle courrait à tout instant le risque d'assimiler l'cuvre au procès-verbal des opérations compositionnelles qui présidaient à son établissement, la privant de la sorte de la possibilité d'être reçue esthétiquement.

Dans quelle mesure l'approche de la genèse d'une cevre à partir des traces matérielles qu'elle laisse ne contredit-elle done pas l'idée d'une réception esthétique? Comment, à partir des données réelles, restituer au fait musical un devenir, pour autant qu'il le mette en scène?

Je me contenterai dans le cadre très réduit de cette contribution de suggérer que le devenir de la musique pose non seulement la question de son aspiration au rang d'objet historique, la question de son historicité, mais également celle de sa dynamique langagière spécifique. Si l'on considerre que «l'art moderne favorise la connaissance gènétique des formes», qu'il constitue une "formulation de notre expérience dynamique», 5 on notera donc également qu'il met en scène la part vive du langage, qui est simultanément un devenir et une inquièude portant sur le devenir. L'intérèt ne serait pas tant de décrire comment l'on aboutit à l'ceuvre, ni même de décrire comment l'œuvre est ou peut ètre reçue, servir de prétexte à une interprétation plìlosophique ou à une mise en perspective historique traditionnelle, mais bien de saisir de quelle manière le devenir de l'cuvre, que l'on peut approcher sur la base des données génétiques, nous informe sur les possibilités d'une expérience esthétique.

4 Theodor W. Adorno, «Fragment sur les rapports entre la musique et le langage» in Quasi una fantasia, pp. 4 et 8 .

3 Hugues Dufourt, «Le Déluge, Philosophie de la musique moderne» in L esprit de la musique. Essais d'esthetigue et de philosophie, Paris, Klincksieck, 1992, p. 23. 